\title{
Secure And Policy Update Method on Big Data Access In Cloud Storage
}

\author{
${ }^{1}$ Shrikant Malge, ${ }^{2}$ Prof.Snehal D. Chaudhary, ${ }^{3}$ Priyanka Paygude \\ ${ }^{1}$ M.Tech. Student, Bharati Vidyapeeth Deemed University College of Engineering BVUCOEP, Department of \\ Information Technology, Pune, India \\ ${ }^{2,3}$ Assistant Professor, Department of Information Technology, Bharati Vidyapeeth Deemed University College \\ of Engineering, Pune, India
}

\begin{abstract}
Big data is key force of modernization diagonally in both educational as well as business. In the Cloud computing, big data security is a current and critical research topic. Cloud computing plus data storage offers clients among different facilities to accumulate as well as run their big data in third party data handling hubs. When a corporation selects to accumulate data or applications on the open cloud, it drops its capacity to have substantial access to the servers crowding its data. As a consequence, probably hypersensitive data is at risk from aids offensives. This difficulty turns into a concern to firm when considering uploading data on to the cloud. In this paper, we have proposed a new method that facilitates capable access control through dynamic policy that updating for big data in the cloud system. In this we have focused on implementing an outsourced policy for update method which is used for Attribute-Based Encryption systems. This method is evading the broadcast of coded data plus reduces the calculation effort of data holders, via building exploit of the earlier coded data among older access policies. We have also proposed a policy updating algorithms that help to access different types of policies. In the end, we have implemented a capable as well as protected method which permits data holder to ensure that whether the cloud server has reorganized accurately or not.
\end{abstract}

Keywords: Cloud, access control, Secure, Attribute based encryption, big Data.

\section{Introduction}

CLOUD computing is an innovative calculating prototype which is assembled on virtualization, analogous as well as distributed, service computing, plus service-leaning design. In the most recent years, cloud computing has materialized as a most powerful prototype in the engineering business, and also it has involved widespread consideration from all together academic circles as well as business. Cloud computing grip the guarantee of offering computing as the fifth service once the additional four services such as water, gas, electricity, plus telephone. The advantages of cloud computing that contain compact prices as well as assets expenses, which raise equipped effectiveness, elasticity, instant time to sell etc. There are different services leaning cloud computing method has been projected, that contains Infrastructure, Platform, in addition to Software Service. Several profitable cloud computing models has been assembled at dissimilar stages, such as Amazon's S3 and IBM's Blue Cloud are infrastructure service systems, whereas Google Application Engine as well as Yahoo Pig are delegate Platform service systems, and the last Google's Apps plus Sales force consumer Relation Management System are software service systems [1]. Hardly any years,

Identity-Based Encryption method has proposed that is moreover identified as Attribute-Based Encryption. In this method, the uniqueness is observed as a list of expressive characteristics. Dissimilar from the identity based encryption, wherever the encrypt data converter might decrypt the file content if and simply if his uniqueness is accurately the similar as what précised via the decrypt data converter, this method allows the data in decrypted form if there are uniqueness overlies above a pre-list entry among the one précised via data converter and the one goes to decrypt data converter. Still, this type of entry stands method was restricted for scheming additional common system since the entry stands semantic cannot convey a common situation [2]. In this cloud storage is a significant service of cloud computing system which recommends different services for data holders to crowd their data in the cloud system. This original model of data crowding and data retrieve services establishes an enormous test to data retrieve handling. Since the cloud server is not able to trust through data holders, also they cannot depend on the servers to do retrieve handling. Coded content Policy Attribute based Encryption is stared as a most appropriate technology for big data retrieve handling in cloud data storage systems, since it offers the data holder additional straight handling on retrieve policies. In this method, there is a power which is answerable for characteristic organization as well as key sharing. The power is also can be the listing workplace in a academy, the individual source section in a business, and so on. The data holder identifies the retrieve policies as well as coded data to the different policies. Every client will be concerned with an undisclosed key replicating its characteristics. A client can convert the data in coded form only when its characteristics assure the retrieve policies [3]. The worldwide characteristics in the method are categorized into 
dissimilar stages to their significance described in the retrieve handling cloud system. Each client in the system shows a set of characteristics in chain of command. The data owner encrypts a data to users in the system that has a certain set of attributes. The coded text file includes a sort of tree retrieve organization. In organize to convert data in the simple message, clients characteristics in chain of command must convince the tree retrieve organization. The view of code text characteristics based encryption can be measured as the simplification of conventional method wherever every characteristic are in the similar stage [4]. A new cryptographic clarification is called as provable characteristic stands words explore. The explanation permits a data client, whose records assure a data holders retrieve handling policy, firstly to explore above the data holders outsourced coded data, secondly farm out the deadly explore functions to the cloud, and lastly validate whether the cloud has authentically accomplished the explore procedures [5].

A protection representation of characteristics based encryption with demonstrable farm out understandable form through beginning a confirmation input key in the production of the data coded algorithm. Then, methods to translate every characteristic based encryption method with farm out simple form data into characteristic based encryption method among provable farm out simple form data. It evaluated among the novel farm out characteristics based encryption; the provable farm out characteristics based encryption neither enhanced the clients and the cloud server's calculation prices excluding several non central procedures nor enlarges the coded text dimension excluding adding up a mix up cost [6]. A parallel coded text characteristic based encryption method is used to parallelize coded text characteristic based encryption and seaport it to the multi-core structural design equipment. Main presentation blockages like a key organization and encryption or decryption procedure are recognized in addition to increase speed. New different encryption process method is accepted for additional presentation expands [7]. A capable text file chain of command characteristic based encryption method is projected in cloud computing. The coated retrieve organizations are incorporated in a particular retrieve organization, and then the tree structured text files are coded through the incorporated retrieve organization. The coded text file machinery connected to characteristics could be collective through the text files. Thus, equally coded text or data storage plus time price of coded data form are accumulated. Furthermore, this method is shown to be safe below the typical theory [8].

In this paper we have implemented a new method that allows doing capable access control through the dynamic policy updating for big data in the cloud system. An attribute-based Encryption system is used for outsourced policy for updated method. Proposed method is evading the broadcast of coded data with reduces the calculation effort of data holders, with the help of the past coded data amid elderly access policies. Policy updating algorithms is defined for the diverse sets of access policies. At the end we have design a safe method that permits data holder to verify whether the cloud server has reorganized appropriately or not [9].

This paper contributes different characteristics:

- It design the policy for updating problem in attribute based encryption systems, also expand a novel method to farm out the policy changing to the server.

- It aims an open and capable data access control method for big data that will facilitate resourceful active policy for changing.

- It proposes policy for changing algorithms for dissimilar kinds of retrieve policies.

- It accomplish dynamic policy modify for additional protected retrieve of big data through lowest calculation time along with rate.

The following paper is ordered are as follows: We will investigate the earlier different big data handling methods as well as various texts based encryption method in section 3 . We have proposed new Secure and Policy Update method on big data access in cloud storage in section 4. We will explain the cloud system workflow in detail in section 5. Section 6 will illustrate a conclusion and future scope in cloud system.

\section{Background And Motivation}

In the cloud computing, accumulating and handing out big amount of data which needs scalability, liability acceptance as well as accessibility. In this Cloud computing convey the entire these during hardware virtualization. Thus, big data plus Cloud computing are two similar perceptions.

As cloud allows big data to be obtainable, scalable as well as fault handling. Industry views big data as an expensive industry prospect. Like, numerous latest businesses such as Cloud era, Hortonworks, Teradata and so on, have ongoing to spotlight on transporting big data as a check or data Base as a check businesses like Google, IBM, Amazon in addition to Microsoft moreover present approaches for clients to use big data on order. Even though big data resolves numerous existing troubles concerning elevated amounts of data, it is a continually varying part that is at all times in improvement plus that at rest causes several problems. In this paper we will present a number of the methods that address by big data as well as cloud computing. As the quantity of data develops at a quick time, maintaining the entire data is actually expenditure. Consequently, businesses must be capable to make rules to classify the life series in addition to the termination date of data. 
Furthermore, they could classify who uses and through what reason customers' data is retrieved. Since data shifts to the cloud storage, protection as well as confidentiality turns into an anxiety that is the issue of broad investigate.

Big data is nothing but a large ability, large speed, in addition to large range data resources which involve innovative types of handling to permit enhanced termination constructing, impending detection as well as procedure optimization. Because of its large amount plus difficulty, it turns complex to handle big data via on-hand record organization tools. An efficient decision is nothing but accumulate big data in the cloud storage, since the cloud storage has abilities of accumulating big data along with handling large quantity of client retrieve in a capable manner. While handling big data in the cloud storage, the data protection becomes a key issue as cloud servers not able to trust on data vendors. In this survey paper, we have centered on resolve the update policy issues in $\mathrm{ABE}$ organizations, plus we have presented a protected and supportable policy which updates outsourcing scheme.

\section{Related Work}

Shucheng Yu et.al. have presented difficult problem by showing plus imposing retrieving different policies which stands on data characteristics, on the other side, permitting the data holder to assign mainly of the estimation tasks concerned in grained data retrieve power that does not belive on cloud servers with no releasing the fundamental data texts [10]. In this they have accomplish this aim via utilizing plus distinctively joining methods of characteristic stands encryption, alternative re-encryption, in addition to idle re-encryption. Their projected method also has relevant assets of client retrieve advantage privacy as well as client undisclosed input liability. In this pervasive examination, they have presented that the implemented system is enormously proficient also protected below offered protection representations.

Kan Yang and Xiaohua Jia has presented design of an retrieve manage structure for multiple security schemes and also proposed an capable as well as protected multiple security retrieve manage scheme for the cloud data storage [11]. Initially they have designed a resourceful multiple security characteristic stands encryption method which does not need a worldwide security also they have try to maintain any retrieve structure. Then, they have proven that the protection in the arbitrary oracle representation. They have also presented a novel method to resolve the characteristic re-trade difficulties in multiple security characteristics stands encryption methods. In this paper they have presented the investigation as well as replication outcomes illustrates that the multiple securities retrieve power method is capable in addition to proficient.

Kan Yang, Xiaohua Jia and Kui Ren has shown a cloud data storage service that permits data holder to contract out their data to the cloud data storage also that offer the data retrieve to the clients. Since the cloud data server along with the data holder is not the trusted area, the tiny trusted cloud data server are not able depend to impose the retrieve policy. To understand this test, conventional schemes typically need the data holder to convert the data in the code language moreover transport un-coded key input to the approved clients. These different schemes, still, usually engage complex key input organization along with large transparency on data holder. In this paper, they have designed a retrieve power structure for cloud data storage methods that accomplished grained retrieve power stands on a modified Cipher content Policy characteristics stands Encryption scheme [12]. In this paper they have planned process which is called a proficient characteristic retrade practice that to manage with the forceful transform of clients retrieve rights in big level schemes. In this investigation they have shown that the projected retrieve power method is probably protected in the unsystematic oracle representation with the capable to be appropriated into perform.

Dongyoung Koo et.al. Has presented the cloud data storage based on record access service, this is a secure skill that will figure a dynamic advertise in the close to opportunity. Even though there have been many investigation projected concerning with protected data access above coded data in cloud data services, mainly they have centered on presenting the harsh protection for the information hold in a third person area. Still, individual's methods need surprising prices central on the cloud data service supplier that could be a major obstacle to accomplish capable data access in cloud data storage. In this paper, they have proposed a capable data recovery method via characteristic stands encryption [13]. The projected method is finest appropriated for cloud data storage methods among enormous total of record. It offers prosperous articulateness as observes retrieve power with rapid explores among effortless associations of penetrating individuals. The projected method moreover assurance data storage protection with client isolation through the data recovery method.

Vipul Goyal et.al. have developed a novel cryptography scheme for fine-grained distributing of coded data that is called as a Key input Policy characteristic stands Encryption [14]. In this cryptography scheme, coded contents are labeled with the different sets of characteristics and confidential input keys are connected with retrieve organization that handle which coded contents a client is capable to convert the data in simple format. They have expressed the applicability of the system structure to distributing of review record plus transmit coded data. The system structure handles allocation of confidential input keys that includes Hierarchical uniqueness stands on the coded data. 
Taeho Jung et.al. has shown a semi-unidentified benefit direct method that is Anony power which is utilized to categorize not only the data separation, but also the client characteristics separation in reachable retrieve power methods. Anony power disperses the essential ability to border the characteristics escape with thus accomplishes semi ambiguity [15]. In addition, it moreover simplifies the file retrieve power to the benefit power, via which rights of every procedure on the cloud record that can be handled in a fine-grained mode. Consequently, they have shown the Anony power F, which completely stop the characteristics escape plus accomplish the complete ambiguity. In this protection investigation exposed that together of the Anony power and Anony power-F are protected below the conclusion and their presentation estimatation reveal the possibility of the proposed methods.

\section{Proposed System}

In this section we have address the problem of handling the big data on the cloud storage with secure data access, therefore we have proposed a innovative method which permits to accomplished access control during the dynamic policy updating for big data in the cloud computing system. An attribute based Encryption system is utilized for farm out policy for updated method. In this the proposed method is escaping the showing of unreadable format data amid condenses the estimation attempt of data holders, through the aid of the history unreadable format data among old access policies. Policy updating algorithms is described for the varied lists of access policies. Lastly we have intended a protected method that allows big data holder to validate whether the cloud server has restructured properly or not.

\section{System Workflow}

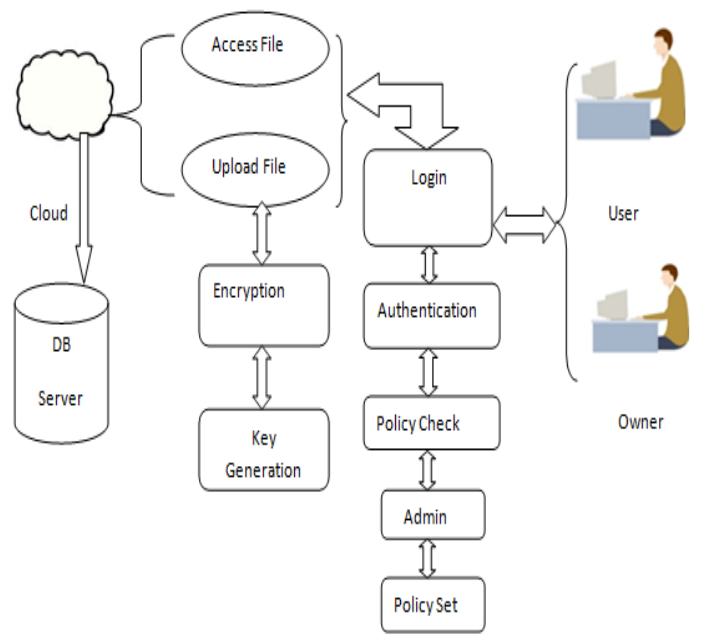

Fig. 1 System Workflow

The proposed method has following different modules to handle big data on the cloud storage are as follow:

\subsection{Authentication Module:}

In this module each authentication of user is not depending on all other plus it is liable for handling the different attributes of users in its area which is nothing but attribute based encryption of the big data. It besides produces a secret public input key couple for every attribute in its area, as well as produces a secret input key for every user depending to his or her different attributes. In this paper we will utilize Attribute Based Encryption for user authentication. And this authentication is completed on user login as well as policy of the admin is also getting inspected. Here user inputs is a user documentation for retrieving text file from the server, Attribute based encryption method is used to confirm that the user is authentication is done or not via verifying its user documentation. Different Policies of the admin are too checked.

\section{Server Module:}

This cloud server module is used to accumulate the big data for data holders along with it offers data retrieve service to different users on the cloud system. This server module is moreover answerable for changing coded contents from previous retrieve policies to latest retrieve policies. The cloud server module is utilized for uploading as well as downloading confidential records on the cloud system. Therefore, whenever users desires to uploading every vital text file he or she requires coded input key. This coded key is created through input key 
creation algorithm. Therefore User will able to retrieve text files or records from cloud server once login to the system.

\section{Owner Module:}

In this module the data owners identify retrieve policies and coded data in these different policies prior to crowding them in the cloud system. They will also request the server module to change retrieve policies of the coded data accumulated in the cloud system. Then, they will also verify that the server module has changed the policies properly or not. Plus holder also require to login for essential retrieve policies plus handling them. In this for coding the data which is accumulated in the cloud server holder requires to receive authorization from the server module.

\section{User Module:}

In this user module every user is allocated through worldwide user uniqueness plus it can generously obtain the coded text files from the server module. This coded text files is not clear to user, therefore user requires to convert this coded text files into simple text files. The user can convert the coded text files, simply when it is attributes assure the retrieve policy identified in the coded text. If the policy of user acquires updated then moreover user should retrieve the data.

\section{Conclusion And Future Work}

In this paper we have implemented an capable method to outsource the policy changing to the cloud server, that can assure every the user necessities. We have also developed an significant attribute-based retrieve control method for big data in the cloud system, as well as considered policy changing algorithms for dissimilar types of retrieve policies. In addition, we proposed a method that allow data holder to verify the accuracy of the coded text changing. In this we will also investigate our method with respect to accuracy, fullness, protection as well as presentation of the system. A one of motivating release crisis for future research work can be accommodation of dynamic data as well as this proposed methods of farm out policy changing can be applied to further attribute based encryption systems.

\section{References}

[1] zhiguo wan, jun'e liu, and robert h. deng, "HASBE: A hierarchical attribute-based solution for flexible and scalable access control in cloud computing”, ieee transactions on information forensics and security, vol. 7, no. 2, april 2012.

[2] Taeho Jung, Xiang-Yang Li, Zhiguo Wan and Meng Wan, "Privacy Preserving Cloud Data Access with Multi-Authorities", 2013 Proceedings IEEE INFOCOM.

[3] kan yang, student member, ieee, and xiaohua jia, fellow," expressive, efficient, and revocable data access control for multi-authority cloud storage ", ieee transactions on parallel and distributed systems, vol. 25, no. 7, july 2014.

[4] Ximeng Liu, Jianfeng Ma, Jinbo Xiong, and Guangjun Liu," Ciphertext-Policy Hierarchical Attribute-based Encryption for FineGrained Access Control of Encryption Data ”, International Journal of Network Security, Vol.16, No.6, PP.437-443, Nov. 2014.

[5] Qingji Zheng, Shouhuai Xu, Giuseppe Ateniese, "VABKS: Verifiable Attribute-based Keyword Search over Outsourced Encrypted Data", IEEE INFOCOM 2014 - IEEE Conference on Computer Communications.

[6] baodong qin, robert h. deng, shengli liu, and siqi ma, "attribute-based encryption with efficient verifiable outsourced decryption", ieee transactions on information forensics and security, 10.1109/tifs.2015.2410137.

[7] Lifeng Li, Xiaowan Chen, Hai Jiang, Zhongwen Li, Kuan-Ching Li, "P-CP-ABE: Parallelizing Ciphertext-Policy Attribute-Based Encryption for Clouds", 978-1-5090-2239-7/16/\$31.00 copyright 2016 IEEE SNPD 2016, May 30-June 1, 2016, Shanghai, China.

[8] Shulan Wang, Junwei Zhou, Member, IEEE, Joseph K. Liu, Member, IEEE, Jianping Yu, Jianyong Chen, Weixin Xie,” An Efficient File Hierarchy Attribute-Based Encryption Scheme in Cloud Computing ”, DOI 10.1109/TIFS.2016.2523941, IEEE Transactions on Information Forensics and Security.

[9] Kan Yang, Associate Member, IEEE, Xiaohua Jia, Fellow, IEEE, Kui Ren, "Secure and Verifiable Policy Update Outsourcing for Big Data Access Control in the Cloud", 10.1109/TPDS.2014.2380373, IEEE Transactions on Parallel and Distributed Systems.

[10] Shucheng Yu, Cong Wang, Kui Ren, and Wenjing Lou, “Achieving Secure, Scalable, and Fine-grained Data Access Control in Cloud Computing”, Technical Program at IEEE INFOCOM 2010. 978-1-4244-5837-0/10/\$26.00 @2010 IEEE.

[11] Kan Yang, Xiaohua Jia, “Attributed-based Access Control for Multi-Authority Systems in Cloud Storage”, 2012 32nd IEEE International Conference on Distributed Computing Systems, 1063-6927/12 \$26.00 @ 2012 IEEE.

[12] Kan Yang, Xiaohua Jia, Kui Ren, "Attribute-based Fine-Grained Access Control with Efficient Revocation in Cloud Storage Systems”, ASIA CCS'13, May 8-10, 2013, Hangzhou, China. Copyright 2013 ACM 978-1-4503-1767.

[13] Dongyoung Koo , Junbeom Hur , Hyunsoo Yoon ," Secure and efficient data retrieval over encrypted data using attribute-based encryption in cloud storage", Computers and Electrical Engineering 39 (2013) 34-46.

[14] Vipul Goyal, Omkant Pandey, Amit Sahai, Brent Waters, "Attribute-Based Encryption for Fine-Grained Access Control of Encrypted Data”, CCS'06, October 30-November 3, 2006, Alexandria, Virginia, USA. Copyright 2006 ACM 1-59593-518.

[15] taeho jung, xiang-yang li, senior member, ieee, zhiguo wan, and meng wan, "control cloud data access privilege and Anonymity with fully anonymous Attribute-based encryption", ieee transactions on information forensics and security, vol. 10, no. 1, january 2015 . 